\title{
Maintenance timetable planning based on mesoscopic infrastructure and the transport service intention
}

\author{
Raimond Wüst ${ }^{1}$, Stephan Bütikofer ${ }^{1}$, Severin Ess ${ }^{1}$, Claudio Gomez ${ }^{1}$, Albert Steiner ${ }^{1}$, \\ Marco Laumanns ${ }^{2}$, Jacint Szabo ${ }^{3}$ \\ ${ }^{1}$ Institute of Data Analysis and Process Design, Zurich University of Applied Sciences ZHAW, \\ 8401 Winterthur, Switzerland, Switzerland, wura@zhaw.ch \\ 2 Bestmile SA, 1007 Lausanne, Switzerland, marco. laumanns@bestmile.com \\ ${ }^{3}$ IBM Research, now at Google Switzerland, Brandschenkestrasse 110, Zurich, Switzerland \\ szabojacint@gmail.com
}

\begin{abstract}
Planners of maintenance intervals and operations have a strong need for rapid development and assessment of comprehensive and reliable timetable scenarios, which are able to satisfy the requirements of both, the train operating company and the infrastructure operating company. To address these requirements, in this paper we present a use case that is based on the 'track-choice and line-rotation' extension of 'PESP', the commonly known model for the generation of periodic event schedules. We call the extended model 'TCFPESP'. This model takes into account the event flexibility requirement of the 'service intention' and makes use of a mesoscopic track infrastructure representation. Both properties support an iterative timetable development process with a 'progressive feasibility assessment', a feature that is requested in practice.

The 'service intention' represents the functional timetable specification. The specification is given by an integrated line concept consisting of a set of lines with data defining their types, frequencies, stop sequence, rotation times and connections. Our proposed model takes functional and operational timetable specifications as input and is applied in an iterative way by changing technical parameters in order to generate a timetable with a feasible capacity allocation. Both, the service intention as well as the mesoscopic infrastructure representation can be configured in the line planning and timetabling system Viriato. This system is widely used by public transport planners and operators. It is therefore possible to configure our timetable model by a standard planning tool.

After the description of the methods developed, we provide a practical proof of concept by testing the use case for different maintenance scenarios. Thereby we can show that, based on the service intention planners are able to quickly develop feasible timetable scenarios for maintenance intervals. The use case presented in this paper refers to generating short-term timetable scenarios but can also be used in long-term strategic planning.
\end{abstract}

Keywords: Periodic Event Scheduling Problem, Mesoscopic railway topology, Service Intention, Timetabling with track assignment 
Declarations of interest: none

\section{Introduction}

\subsection{State-of-the-art}

In the operational management of railway networks, an important requirement is the fast adaptation of timetable scenarios, in which operational disruptions or time windows with temporary unavailability of infrastructure, for instance during maintenance time windows, are taken into consideration. In those situations, easy and fast reconfiguration and recalculation of timetable data is of central importance. This local and temporal rescheduling results in shifted departure and arrival times and sometimes even in modified stop patterns at intermediate stations of train runs. We will refer to this scheduling process as interval planning (IP). In order to generate reliable timetabling results, it is a prerequisite that train-track assignments as well as operational and commercial dependencies are taken into consideration and that all these aspects are not in conflict with each other. Hence, finding the right level of detail for modelling track infrastructure and train dynamics is crucial for supporting the planning process in an optimal way. In recent years, this requirement motivated several research groups to combine common timetabling procedures with constraints resulting from mesoscopic infrastructure information. Some of the most important approaches that are relevant to our work are discussed in the following.

The quality of all timetabling models is strongly dependent on an accurate estimation of running, dwell and headway times given the microscopic infrastructure. Hansen and Pachl (2008) show how these times at critical route nodes and platform tracks must be taken into account for train processing and present an in-depth timetable quality analysis depending on these parameters. Schlechte et al. (2011) presented an algorithmic approach to create a macroscopic infrastructure model from a given microscopic model.

The periodic event scheduling problem (PESP) is a powerful model for periodic schedules introduced by Serafini and Ukovich (1989), which was first applied to train scheduling by Schrijver and Steenbeck (1994). Extensive descriptions of different mathematical modelling approaches, solution algorithms, complexity results and the modelling power for railway timetables of PESP can be found in Caimi (2009), Liebchen and Möhring (2007) and Peeters (2003). The use of a service intention (SI) (see section 1.2) to describe the offered the train services and to parametrize the PESP model was introduced by Wüst et al. (2008) and investigated extensively by Caimi (2009) and by Caimi et al. (2011a).

PESP models normally produce timetables on a macroscopic level without considering track and station capacities. Starting from this (macro) timetable the train timetabling problem (TTP) tries to determine the exact departure and arrival times of these trains respecting the capacities. First results for TTP can be found in Caprara et al. (2002). A broad overview for TTP is given by Schlechte (2012). Bešinović et al. (2016) presented a micro-macro framework based on an integrated iterative approach for computing a microscopically conflict-free timetable that uses a macroscopic opti- 
mization model with a post-processing robustness evaluation. Caimi et al. (2011b) extend PESP by proposing the flexible periodic event scheduling problem (FPESP), where intervals are generated instead of fixed event times. By applying FPESP, the output does not define a final timetable but an input for finding a feasible timetable on a microscopic level (Caimi (2009) and Caimi et al. (2011a)). De Fabris et al. (2014) introduced a mesoscopic timetabling model which simplifies the representation of station layouts to combine fast computation of macroscopic models with the accuracy of microscopic models. In our modelling approach we also refer to a mesoscopic representation of the infrastructure in order to assess operational feasibility of the timetabling results.

\subsection{Research goals}

In this article we present solutions for two research goals: (i) We develop a method for the timetable generation based on an innovative timetabling model 'TCFPESP' that considers mesoscopic infrastructure and the transport service intention (SI); (ii) From the future SBB timetable planning process (see section 2.1) we derive a use case for interval planning, which utilizes the method mentioned in (i) for timetable generation.

Next to the proposed model, the main contribution of this paper consists of a use case including an iteration scheme for applying the timetable method with slightly changing objectives for timetable generation. The necessary data structures can be managed in a standard timetable editor. Finally, the use case is applied in a practical case study.

Similar to Caimi et al. (2011b), our modelling approach for the timetable generation is also based on an extension of the periodic event scheduling problem (PESP) and takes the SI as input data structure. The SI was first described in Wüst et al. (2008), formally specified in Caimi (2009) and integrates commercial timetabling requirements given by the respective line concept on the one side and technical constraints on the other. The 'line concept' represents functional timetabling requirements based on a set of lines with data defining their types, frequencies, train paths, rotation and separation times and connections. In order to preserve acquired knowledge about customer flows for the subsequent planning step, customer transfers between lines at specific stations are also included in the SI. In accordance to de Fabris et al. (2014), we call our level of abstraction of the available resources 'mesoscopic topology'. Along with the functional requirements of the SI, this mesoscopic infrastructure data model of a given scenario is entered via a standard timetable editor (see, e.g., SMA Viriato (2018)). We demonstrate the detailed sequence of the planning actions that planners must execute in order to generate timetable results for different scenarios of maintenance intervals for the interval planning (IP) use case.

\subsection{Structure of this paper}

This article is structured as follows: In section 2, we describe the methodology for achieving the research goals mentioned in section 1.2. In section 2.1 (IP business requirements) we provide a short description of the special business requirements of 
interval planning, which are based on the future SBB process model for timetable generation. Based on these requirements we propose in section 2.2 (Method for computer aided interval planning) an IP use case for computer aided interval planning which is based on the SI and the mesoscopic infrastructure. To validate the proposed method, we present a network segmentation method in section 2.3 (Network segmentation). We apply this method to isolate a partition of the SBB rail network for testing the IP use case in a scenario of suitable size. In section 2.4 (Method for generating traffic plan with flexibility in IP) we provide a detailed description of the IP use case based on two iteration schemes. In section 2.5 (Generation of traffic plan based on a standard planning tool ) we describe how data that is required as input in the proposed use case can be handled in a standard planning tool like Viriato. In section 3 (Case study 'Kerenzerberg') we present the results of applying the methods introduced in section 2 as well as their interdependencies in a real-world test scenario. The section contains a detailed description of the actions belonging to the proposed use case and to the application of the proposed method. In section 4 we conclude with a summary of the encouraging results of the presented case study. We consider them as a proof of concept for our proposed use case. Finally, we provide a brief outlook on future work.

\section{Methodology}

One of the most important requirements for public transport services is its usability compared with competing transport modes. Two factors have a significant impact on the usability. The first one is the aspect of the regularity or periodicity of a timetable, which allows travellers for easily remembering departure and arrival times and hence making travel planning much simpler, especially for regular travellers. The second aspect deals with the integrated transport chain. The transport chain is characterized by changing transport modes between local (de-)feeding lines like bus or streetcar lines and high-performance train lines with higher speeds and capacities. Of course, this includes the change between different line types (e.g. far distance line, commuter line) or lines as well. Here the realization of short connection times is the main objective. The set of relevant transport chains can be obtained by combining origin-destination-demand matrices with potential line pools in the line planning process step (see Figure 1, 'line planning, line concept', Schöbel and Scholl (2006) and Friedrich et al. (2017)). The integrated information regarding lines and transport chains represents the intended transport service and is called service intention (SI). Both are important usability aspects and can be realized technically by introducing a countrywide integrated fixed interval timetable (IFIT) (see for example Herrigel (2015) for an explanation of the fundamental idea), which synchronizes the service schedules of almost all carriers. The integration of services of different TOCs requires a highly iterative process of timetable generation. This process will be explained in the following section.

\subsection{IP business requirements}

At the beginning of the collaboration project with our industry partner SBBInfrastructure, business analysts reviewed the planning process resulting in a descrip- 
tion of the streamlined future timetable generation process, which is summarized in Figure 1. In order to define business requirements for a timetabling method that allows to achive the research goals (i) and (ii), described in section 1.2 we first describe the aim of each step in the future timetable generation process. This is necessary, because the timetable generation process is supposed to be executed in the context of strategic global timetable generation (long term) as well as in the context of minor local operational adaptations of an existing timetable (short and medium term). Such an operational timetable adaptation is required in the context of IP. We concluded that the definition of a suitable interface between line planning and timetabling is an important issue and a central part of the business requirements. With the iteration schemes described in sections 2.4.1 and 2.4.2 we show that the SI concept satisfies the requirements for the interface between the line planning and timetabling process step shown in Figure 1. In this section we will explain the process steps of Figure 1 in more detail.

The desired transport service is based on requests from various stakeholders and is consolidated by the TOCs. This functional description is represented by the SI. The functional requirements represent scenarios of transport chains, which have been consolidated before. In the first set of process steps, the SI is defined by the involved TOCs and translated into a capacity requirement, mapped onto railway lines and stations. It can be visualized in terms of net graphs, line diagrams and passenger-flow tables (see TOC 'Passenger and Freight Assignment' and 'line planning, line concept' in Figure 1). In the second set of process steps, the SI's of the different TOCs and the capacity requirements of the different train lines must be consolidated and checked for operational feasibility. This is done by the integrating IOC. SBB calls these process steps 'traffic planning' and 'capacity planning' (see Figure 1). The result of process step 'traffic planning' corresponds to the consolidated SI. In the scope of this paper we will call the consolidated SI 'commercial timetable'. The commercial timetable is the basis for the communication of the timetable to the customer. Additionally, the consolidated SI requires a feasibility check based on the following process step 'capacity planning'. The result of this process step is a validated version of the service intention, which accounts for capacity constraints defined by track occupation, headway, transfer and line rotation time requirements. In addition, constraints resulting from maintenance and construction requirements are accounted for. All these aspects of capacity consumption are integrated in the capacity plan, which we will call 'timetable with event flexibility'.

This process model assumes a close cooperation between the different Train Operating Companies (TOC) and the Infrastructure Operating Company (IOC), including (to a certain degree) a barrier free data access for the IOC. This improved data transparency is needed to insure a specified service level that holds also in case of IP or operational disruptions.

As one can see in Figure 1, in cases of reduced capacity (compared to resource conditions of the standard timetable), the IOC has the responsibility of providing the best service quality possible. That means that in the use case of interval planning or operational disruption, the IOC must have access to demand and service specific data (managed by the TOCs) which determine the input for the process of generating a consolidated SI. 


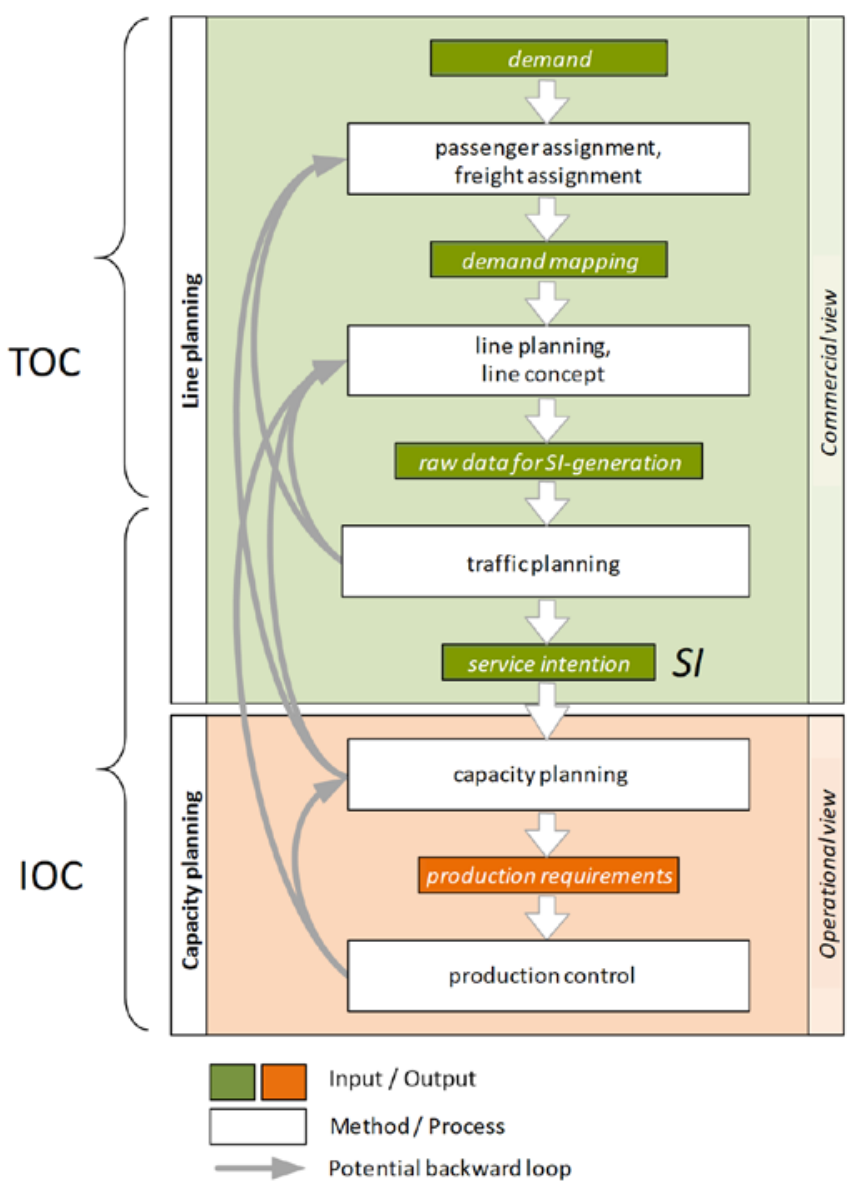

Figure 1. The SBB timetable planning process: overview and integration of line planning into the timetable planning model. The grey arrows in the figure demonstrate that when going back in the in the process chain, data referring to the operation of train lines and passenger demand have to be shared by TOC and IOC. Further explanations are given in the text. Adaptation from Howald et al. (2017)

Figure 1 also shows that the planning process is an iterative one, indicated by the grey backward loop arrows. During this process iteration the level of detail of the resulting plan is progressive because under conditions of long- and medium-term timetable planning, knowledge and decisions regarding functional (commercial) and non-functional (technical and operational) timetabling requirements are getting more concrete. In case of reduced resource availability resulting from short term operational disruptions or maintenance work, this may cause timetable conflicts that can only be resolved by relaxing the functional timetabling requirements. Hence, the SI has to be adapted temporarily.

With the methods that will be presented in the following sections, we attempt to operationalize the SI based planning step (Figure 1: 'traffic planning') in terms of the 
IP use case and the proposed method for computer aided interval planning based on mesoscopic infrastructure and the transport service intention.

\subsection{Method for computer aided interval planning}

The generation and investigation of feasible event times for individual train runs and corresponding resource allocations fitting into the structure of an IFIT is usually done manually. For this reason, timetabling is considered a time consuming and challenging task even for experienced planners. On the other side, algorithmic approaches for solving this task computationally require models based on microscopic information about track capacity, like discussed, for instance in Bešinović et al. (2016) or, in an intermediary step, by defining possible train routes as outlined in de Fabris et al. (2014), from which headway constraints for trains can be derived. We present a generic approach for setting headway constraints and other operational dependencies like turnaround times, and the service intention, which makes use of the mesoscopic infrastructure. The corresponding data are implemented and managed in a standard timetable planning system like Viriato (see, e.g., SMA (2018)).

\subsubsection{Mesoscopic infrastructure model}

To illustrate the level of detail of the respective infrastructure mapped onto a mesoscopic topology we refer to an example of the SBB "Grobkonzept Linienplanung” in Howald et al. (2017, see Figure 2a). The mesoscopic topology consists of operation points linked by route-sections. At each operation point and route-section there is a given number of tracks. Each location that provides an option to change tracks is assigned to a new operation point. If there are customer services assigned to an operation point, it is classified as 'commercial', otherwise it is classified as 'operational'. In our topology model, we introduce graph nodes for both operation points and route-sections connecting two operation points. The capacity ' $\mathrm{C}$ ' of each node is defined by the number of enumerated tracks of the operation point (see Figure $2 \mathrm{~b}$ ). The connectivity of the tracks at each node are additional node attributes and can be configured in Viriato (see Figure 7). From this node topology we derive our event activity network representing all potential track specific event dependencies (see section 2.2.2).

Timetables that are mapped to mesoscopic infrastructure enable a much better feasibility assessment of the result compared to considering only the macroscopic infrastructure. On the other hand, the gap in the level of detail to microscopic infrastructure in terms of feasibility assessment can be reduced substantially, if the event times that are assigned to mesoscopic topology nodes are within a certain range of flexibility (see Caimi (2009) and Caimi et al. (2011b)). We make use of the mesoscopic topology together with the event flexibility according to the FPESP model, introduced in section 2.2.3. This allows generating periodic timetables with a reasonably good assessment of feasibility. The model generates results with flexibility to find a conflict free resource allocation taking a micro-topological level of detail into consideration in a subsequent planning step (Figure 1: 'production control') or if planning has to account for slightly different individual conditions, for example during the course of a day or to consider the operational variability. 


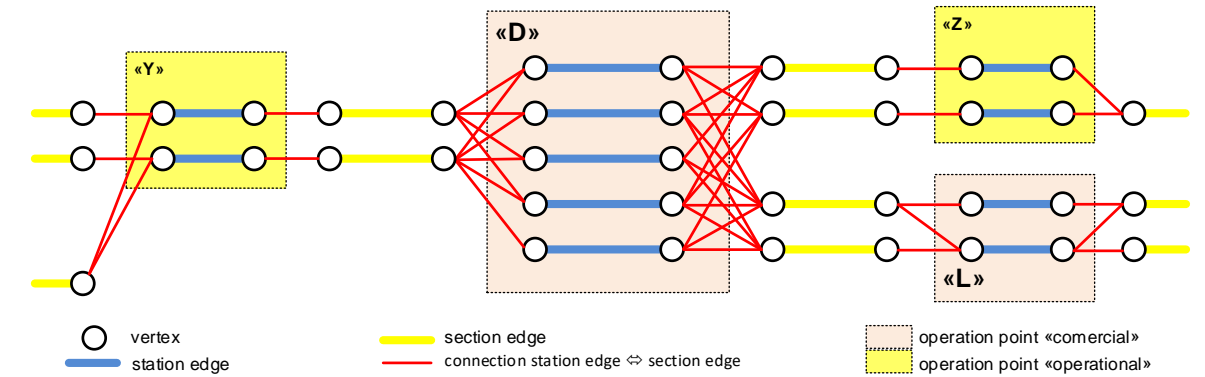

b

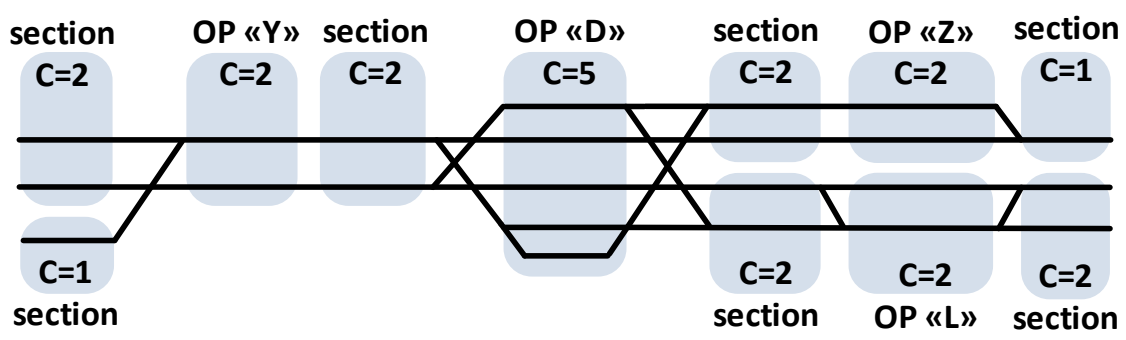

Figure 2: a) Mesoscopic infrastructure example from the SBB “Grobkonzept Linienplanung” in Howald et al. (2017). b). Extracted topology information: Each operation point, and each linking track segment is mapped into a graph node, represented by a grey shaded box. The node attribute ' $C$ ' indicates the track capacity of each node. Switches between node tracks allow for changing tracks when moving from one node to the other.

\subsubsection{Event activity network and periodic timetabling}

The event-activity network (EAN) is the input for our timetable model. It is constructed based on mesoscopic infrastructure information and the SI.

The SI is defined by a set of train runs. Each train run belongs to a line $L$ and is characterized by the sequence of sections that are traversed and a corresponding time interval, which is required for either running or stopping on a corresponding track section. Each time interval has a minimal and maximal value. Stop nodes typically provide a service for boarding or de-boarding a train. Together, a pair of train runs moving in opposite directions makes up a train circulation.

The mesoscopic infrastructure consisting of sections is summarized as a set $I$ of operation points. Operation points are largely tracks and stations but can also be other critical resources as junctions (see example below). As mentioned before, each operation point $i \in I$ is associated to a capacity consisting of a set of tracks $T_{i}$. A train run $l \in L$ is described by a sequence of operation points of $I$.

Based on our mesoscopic model we algorithmically create an event-activity network $(E, A)$. The set $E$ of events consists of an arrival event $\operatorname{arr}_{l i}$ and a departure event $\operatorname{dep}_{l i}$ for each train run $l \in L$ and operation point $i \in l$. The activities $a \in A$ are directed arcs from $E \times E$ and describe the dependencies between the events. For every train run we have arcs between arrival and departure events at the same operation points (dwell times or trip times) and arcs between departure and arrival events of successive operation points (time needed for the travel between operation points). Further arcs include connections between train runs, headways and turnaround opera- 
tions (see section 3). Connections and turnaround information are given in the SI. Headways are derived from the mesoscopic infrastructure and the train runs in the SI. We refer to Liebchen and Möhring (2007) for a detailed overview of the modelling options of dependencies. Figure 3 provides a sample of such an event-activity network.

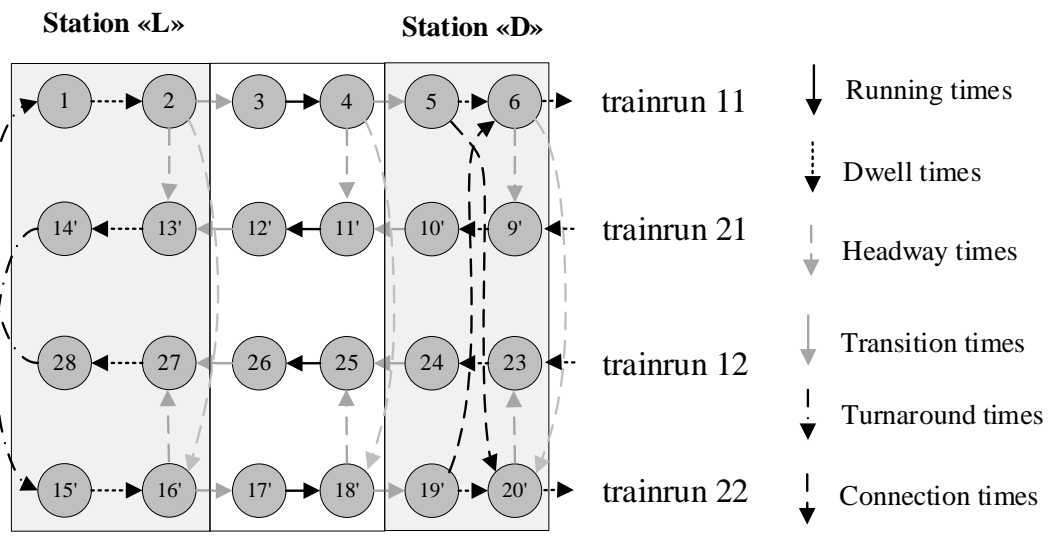

Figure 3: Sample of an event-activity network, where arcs connect arrival and departure events. Nodes belonging to grey shaded boxes indicate events at operation points (here Station «L» and Station «D»). Other nodes indicate track type arrival and departure events. Arrow line styles indicate different types of time dependencies.

Headway arcs $a \in A_{H}$ are especially important for explaining the timetable model below. Headway arcs are used to model safety distances between trains running in the same and in opposite directions (see example in Figure 3). For the sake of simplicity we consider in the formal description of our timetable model in section 2.2.3 only headways related to one operation point, i.e., we omit headways for train runs in opposite directions over several successive operation points. These headways can be easily included in the event-activity network. They are included in our implementation of the timetable model.

The classical PESP model tries to determine a periodic schedule on the macroscopic level (i.e. without using the tracks at an operation point) within a period $\mathrm{T}$. Event $e \in \mathrm{E}$ takes place at time $\pi_{e} \in[0, T)$. The schedule is periodic with time period $T$, hence each event is repeated periodically $\left\{\ldots, \pi_{e}-T, \pi_{e}, \pi_{e}+T, \pi_{e}+2 T, \ldots\right\}$.

The choices of the event times $\pi_{e}$ depend on each other. The dependencies are described by arcs $a=(e, f)$ in $A$ and modeled as constraints in PESP. The constraints always concern the two events $e$ and $f$ and define the minimum and maximum periodic time difference $l_{a}$ and $u_{a}$ between them. These bounds are given as parameters in the PESP model. We therefore look for the event times $\pi_{e}$ for every $e \in E$ that satisfy all constraints of the form

$$
l_{a} \leq \pi_{f}-\pi_{e}+p_{a} T \leq u_{a},
$$

for all $a=(e, f) \in A$, where $p_{a}$ is an integer variable that makes sure that these constraints are met in a periodic sense. 


\subsubsection{Periodic Timetabling with Event Flexibility}

In order to avoid tedious iterations between the process steps "microscopic capacity planning" and "mesoscopic capacity planning” in case of infeasibility of the microlevel problem, one can improve the chance of finding a feasible solution by enlarging the solution space in the micro-level. This approach has been described in detail in Caimi et al. (2011b). We also implement this event flexibility model by (optionally) adding some flexibility for the events of the EAN by introducing lower and upper bounds to the event times of the arrival and departure nodes in Figure 4. The final choice of the event times in the range between the lower and upper bound shall be independent for each event such that each value of the end of an activity arc should be reachable from each time value at beginning of that activity arc.

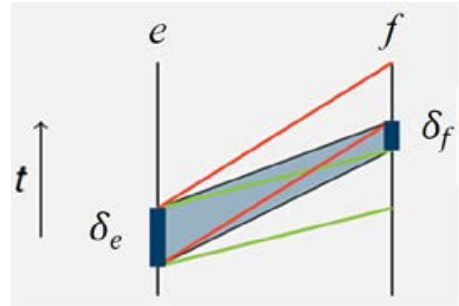

$\mathrm{b}$

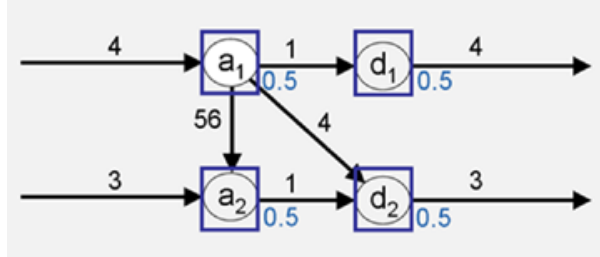

Figure 4: Target oriented placement of time reserves (adapted from Caimi (2011b)): a) Time frames $\left[\pi_{e}, \pi_{e}+\delta_{e}\right]$ in place of time points $\pi_{e}$. By implementing this method, the normal PESP constraints $l_{a} \leq \pi_{f}-\pi_{e}+p_{a} T \leq u_{a}$ now becomes $l_{a}+\delta_{\mathrm{e}} \leq \pi_{f}-\pi_{e}+p_{a} T \leq u_{a}-$ $\delta_{f}$ (see section 2.2.4). b) In this EAN example this means that instead of planning time points $\left(\pi_{a_{1}}, \pi_{d_{1}}, \pi_{a_{2}}, \pi_{d_{2}}\right)$ we plan time frames $\left[\pi_{e}, \pi_{e}+0.5\right]$ for $e \in\left\{a_{1}, d_{1}, a_{2}, d_{2}\right\}$.

We are not forced to add this flexibility to all the events, but we can select the nodes where we want to add it based on user defined rules, for instance only nodes corresponding to events in a main station area with high traffic density, where it is more difficult to schedule trains on the microscopic level. In general, one can say that this placement of flexibility is the timetable configuration feature that has the highest impact on improving operational stability. For our proposed timetabling model, we integrate an extended PESP model (based on mesoscopic infrastructure, see section 2.2.4) with the "flexible PESP" (FPESP) model in order to generate timetables with event slots on a mesoscopic level. For more details regarding the FPESP model, we refer to the article of Caimi et al. (2011b).

\subsubsection{Track-choice PESP model with event flexibility.}

In order to satisfy the practical requirement for assessing the feasibility of a timetable scenario in terms of available track capacity, we extend the classical PESP resp. FPESP model by using the number of tracks $T_{i}$ at each operation point $i \in I$. We will refer to this extended model as the track-choice FPESP model (TCFPESP). It assigns the arrival event $\operatorname{arr}_{l i}$ and the departure event $d e p_{l i}$ of train run $\mathrm{l}$ at operation point $i$ uniquely to a track in $T_{i}$. We can use these assignments to switch on headway arcs 
$a \in A_{H}$ by using a big-M-approach.

In addition to the variables $\pi$ and $p$ from the PESP model we need:

(i) Binary variables $t c_{e t}$ (track choice) for each event $e \in E$ and track $t \in$ $T_{i(e)}$, where operation point $i(e)$ is associated to event $e$, i.e. $e$ is equal to $\operatorname{arr}_{l i}$ or $d e p_{l i}$ for a train run $l$.

(ii) Binary variables $h_{a}$ for every headway edge $a=(e, f) \in A_{H}$. Headway edges are always between events at the same operation point, therefore $T_{i(e)}=T_{i(f)}$ holds.

(iii) Positive variables $\delta_{e}$ for each event $e \in E$ to model the event flexibility.

The TCFPESP model is then defined by:

TCFPESP model: $\min f(\pi, p, \delta)$

$$
\begin{array}{cl}
\text { s.t. } \quad l_{a}+\delta_{e} \leq \pi_{f}-\pi_{e}+p_{a} T \leq u_{a}-\delta_{f}, & \forall a=(e, f) \in A \backslash A_{H}, \\
l_{a}+\delta_{e}-\left(1-h_{a}\right) M \leq \pi_{f}-\pi_{e}+p_{a} T & \forall a=(e, f) \in A_{H}, \\
\leq u_{a}-\delta_{f}+\left(1-h_{a}\right) M, & \\
\sum_{t \in T_{i(e)}} t c_{e t}=1, & \forall e \in E, \\
t c_{a r r_{l i} t}=t c_{d e p_{l i} t}, & \forall l \in L, i \in l, t \in T_{i}, \\
h_{a} \geq t c_{e t}+t c_{f t}-1, & \forall a=(e, f) \in A_{H}, t \in T_{i(e)} \\
t c_{e t}, h_{a} \in\{0,1\}, \pi_{e} \in[0, T), p_{a} \in \mathbb{Z}, \delta_{e} \geq 0, & \forall e \in E, t \in T_{i(e), \quad a \in A,}, \quad
\end{array}
$$

where $M$ is a big enough natural number.

In (1) the normal FPESP constraints are summarized (without headway arcs). (2) defines the headway constraints, which can be switched off with a big-M technique. The assignment of the events to the tracks is done in (3). (4) is used to assign the corresponding arrival and departure events to the same track. In (5) the headway variable is set to 1 , if the events take place on the same track, i.e. the headway is required at this operation point.

There are many different objective functions $f(\pi, p, \delta)$ described in literature (see Liebchen and Möhring (2007) for the general PESP model and Caimi et al. (2011b) for the FPESP model). In our test case below we use two objective functions.

\section{Objective Functions:}

- MINTRAVEL: We minimize all passenger relevant times (i.e. $t \in A_{T}$ the set of trip arcs, $d \in A_{D}$ the set of dwell arcs and $c \in A_{C}$ the set of connections times). The weights $w_{t}, w_{d}$ and $w_{c}$ can be used for prioritizing certain times, e.g. connection times. The objective function $f_{T T}$ is defined as 


$$
f_{T T}(\pi)=\sum_{t \in A_{T}} w_{t} \Delta_{t}+\sum_{d \in A_{D}} w_{d} \Delta_{d}+\sum_{c \in A_{C}} w_{c} \Delta_{c}
$$

where $\Delta_{\mathrm{a}}=\pi_{f}-\pi_{e}$ for an arc $a=(e, f)$ holds. According to Caimi et al. (2011b) we will call the TCFPESP model with this objective function MINTRAVEL.

- CONTRAVEL: We maximize the flexibility in a given range at certain arrival and departure events. The objective function $f_{\text {flex }}$ is defined as

$$
f_{\text {flex }}(\delta)=\sum_{e \in V} w_{e} \delta_{e}
$$

where $V \subseteq E$ is the set of all events where flexibility is introduced. Furthermore, we add two constraints. The passenger travel time has to be smaller than $(1+\epsilon)$ times the best possible travel time from the model MINTRAVEL. The flexibility for all events is bounded by a maximal flexibility $\delta_{\max }$ for a better distribution of the flexibility to all events. The two constraints are given by

$$
f_{T T}(\pi) \leq(1+\epsilon) f_{T T}^{*} \text { and } \delta_{e} \leq \delta_{\max } \forall e \in E,
$$

where $f_{T T}^{*}$ is the optimal value found for $f_{T T}$ in the MINTRAVEL model. We will call the TCFPESP model with the objective function in (1) and the additional constraints in (3) CONTRAVEL according to Caimi et al. (2011b). $\epsilon$ is a parameter controlling the quality of the schedule for the passengers' travel times and the weights $w_{e}$ can be used for individual adjustments in event flexibility to maximize timetable robustness.

Both models MINTRAVEL and CONTRAVEL are mixed integer linear problems. In this paper all the weights in the objective functions of MINTRAVEL and CONTRAVEL are set to 1 . In this way the event flexibility is distributed uniformly for all events. In Wüst et al. (2019) the authors demonstrate that if the delay sensitivity and delay impact of each timetable event are evaluated iteratively, these weights can be adjusted in a suitable way to improve the overall timetable robustness. We provide further details about the implementation and the size of these models in section 3. The TCPESP variant of the model (i.e. without event flexibility) has been presented recently in Wüst et al. (2018).

\subsection{Network segmentation}

In order to avoid putting too much effort into entering information that is not needed and rather focus on the relevant perimeter for the IP timetabling scenario, one has to identify which part of the entire railway network has to be accounted for. The relevant lines and services operating on the subnetwork, which will be affected by the construction or maintenance sites, have to be identified in a first step. In a second step, those lines, which are coupled (e.g. by transfers or technical dependencies) to these affected lines have to be found. 
In the second step, one has to identify the sub-network nodes which isolate the relevant infrastructure segments from the irrelevant periphery. In this way one obtains a disaggregated subnetwork containing the relevant infrastructure segments and an aggregated subnetwork, representing infrastructure on the macroscopic level (see dashed square area on the top of Figure 5a). The disaggregated subnetwork is configured with all mesoscopic details. On this disaggregated subnetwork all train movements are planned in detail for every single IP-scenario. For each line coming from or going beyond the boundary nodes of the disaggregated subnetwork we create a virtual end station node which is connected by a single section to the corresponding boundary node. The section lengths with the appropriate trip times, the turnaround times of the line outside the disaggregated subnetwork together with the run- and dwell times within the disaggregated subnetwork have to sum up to the proper roundtrip time. This segmentation of disaggregated subnetwork and aggregated subnetwork into a new mesoscopic infrastructure model is illustrated in Figure 5b.

a
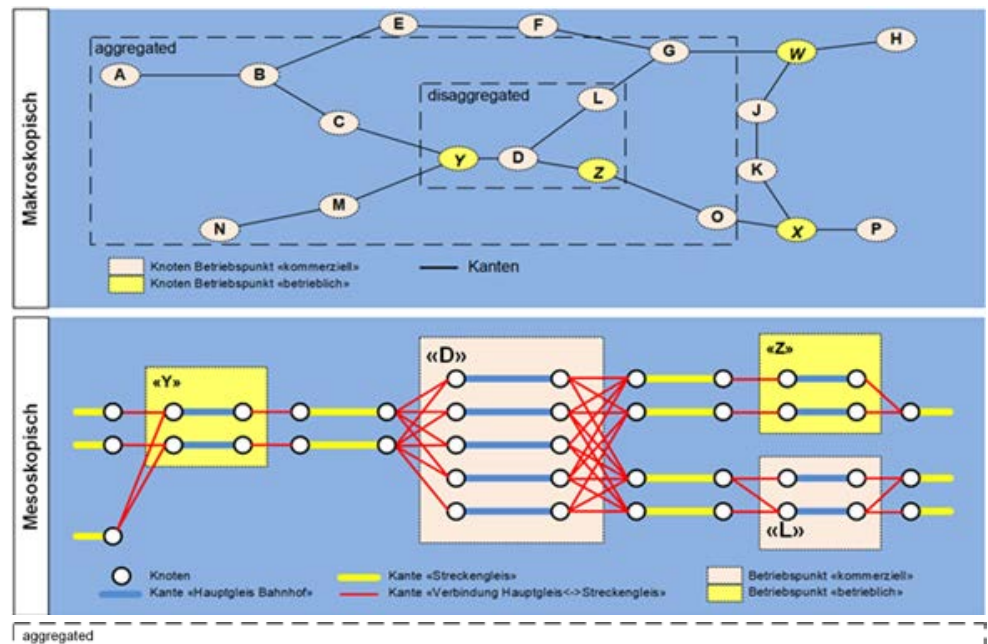

b

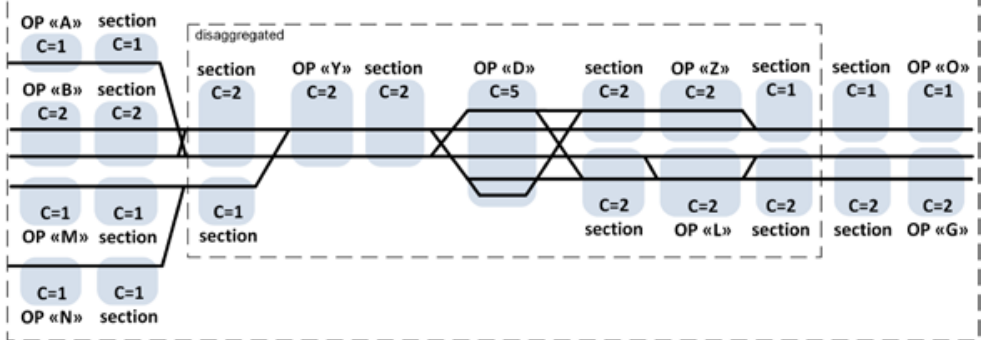


Figure 5: a) Example of mesoscopic and macroscopic topology from Howald et al. (2017). In order to divide the relevant infrastructure for the IP timetabling scenario into a segment with the relevant level of detail and a peripheral part with more coarse information, the railway network is partitioned into subnetworks with different topology levels. A disaggregated subnetwork containing the relevant infrastructure segments on mesoscopic level and an aggregated subnetwork, representing infrastructure on the macroscopic level. b) The figure illustrates the disaggregated subnetwork representation in our model which can be configured with detailed mesoscopic information.

\subsection{Method for generating a timetable with event flexibility}

In the IP use case a timetable with event flexibility, including track assignment, is generated, if possible. In this section we will describe the implementation and the software used in detail. First, we describe the actions of the IP use case from the point of view of a planner (see Figure 6):

1. In the first step, the planner has to enter SI data into the planning system (Viriato).

2. After the scenario configuration in Viriato, a Viriato-database extract is used to add additional SI data (not implemented in Viriato) like transfer times between lines, turnaround times and time dependencies which implement e.g. service frequencies. These additional data are entered to a data mask of the ZHAW planning tool (in-house development based on the ' $\mathrm{R}$ '-development software, R (2019)).

3. The planner initiates the timetable calculation with the TCFPESP model for the IP-use case by selecting the appropriate objective function and parameters according to the iteration schemes of sections 2.4.1 and 2.4.2 below. In the ZHAW planning tool, this induces the building of the EAN and the data import to the Algebraic Modeling System GAMS (2018). The TCFPESP model is implemented in GAMS. GAMS attempts to automatically calculate a solution based on the chosen model with a MIP solver (we use CPLEX 12.6.3).

A feasible solution is then returned to Viriato. If no feasible solution can be generated, the planner has to review and eventually relax the SI. This may lead to a backward loop to 'line planning, line concept' in Figure 1. 


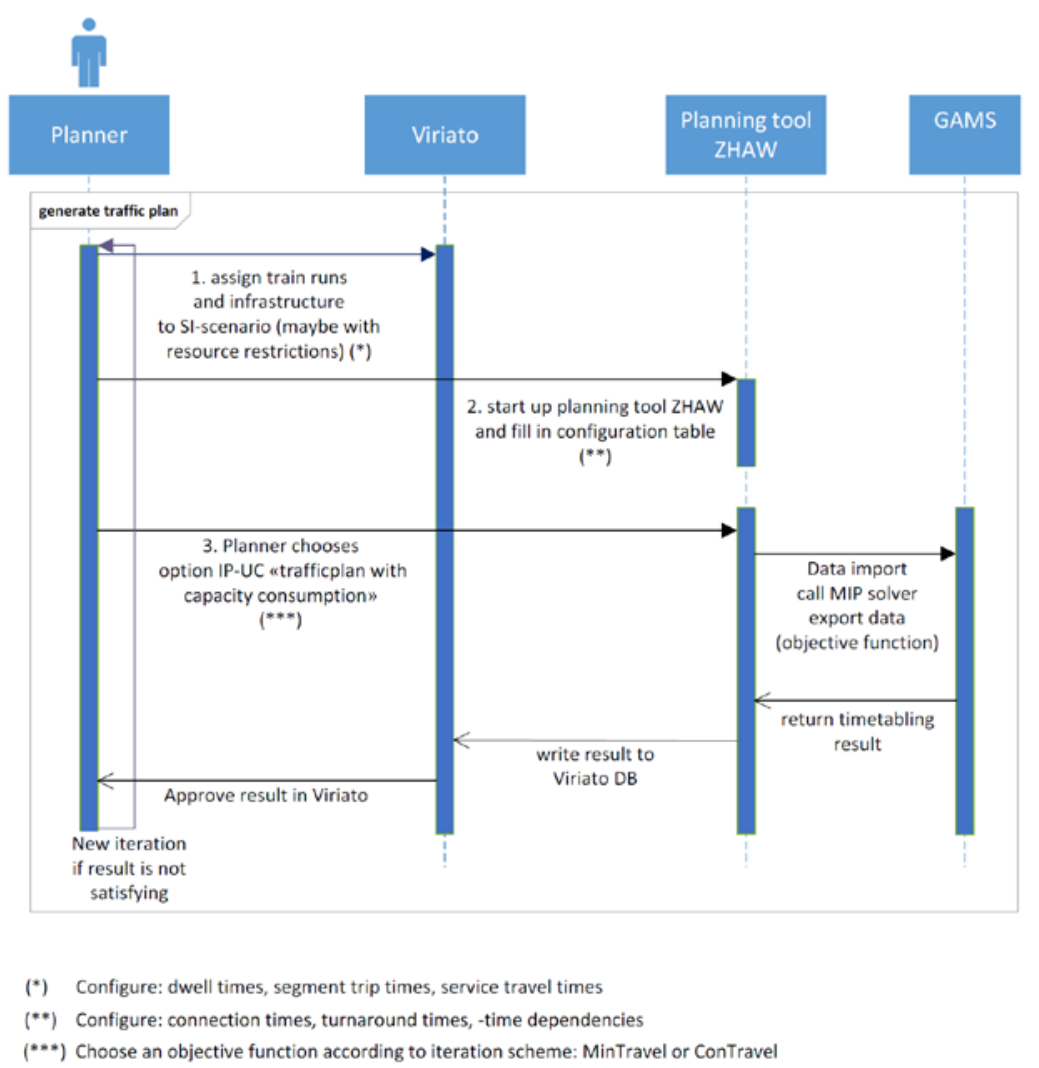

Figure 6: Sequence diagram and system functions of actions during the use case IP. The sequence diagram shows the tasks and functions of the involved planner and system components. Next to the planner there are several system components involved. Viriato (see SMA (2018)) is used as timetable data editor. The planning tool ZHAW implements all timetable data preparation functions not included in Viriato. The main actions within this use case are executed according to the explanation in the text and the iteration schemes described in section 2.4.1. The TCFPESP-model is solved in GAMS. For GAMS see GAMS (2018)

In the following two sections, we describe the methods for the construction of a timetable with event flexibility under normal (unrestricted) operational conditions and under restricted conditions due to maintenance.

\subsubsection{Construction of a timetable with event flexibility for periods with normal operations}

The heuristic iteration scheme 1 is the standard iteration scheme for applying TCFPESP (see section 2.2.4) under normal operations. If we are able to compute a timetable with iteration scheme 1 , we refer to it as reference timetable. If the availability of the involved resources during interval planning is restricted compared to the 
reference timetable, a new (temporary) interval timetable must be generated with the help of the heuristic iteration scheme 2 in section 2.4.2, below.

Aim: Try to generate a feasible reference timetable with event flexibility. Input:

- $\quad$ SI-data (Line data, line transfers, time dependencies, track infrastructure, rolling stock, train properties, etc.)

- Maximal size of flexibility $\delta_{\max }$ for all arrival and departure nodes (available for planning on the micro-level or for stability reasons)

- $\quad$ Parameter $\epsilon$ for controlling maximal deviations of optimal passenger travel times

- $\quad$ Bound on rolling stock per line

(at the beginning the sizes of the event flexibility $\delta_{\max }$ and parameter $\epsilon$ are set to default values and adapted during the iteration in order to achieve the feasibility or improve the stability of the timetable scenario)

\section{Iteration scheme 1}

1. Solve the model MINTRAVEL. We get a timetable with best possible travel times $f_{T T}^{*}$. If the model MINTRAVEL is not feasible adjust SI and go to step 1.

2. Compare the necessary amount of rolling stock per line for the timetable from the MINTRAVEL model with the given bound on rolling stock. If one line needs too much rolling stock, adjust SI and go to step 1.

3. Solve the model CONTRAVEL, allow passenger travel times to be maximal $(1+\epsilon) f_{T T}^{*}$. We get a timetable with (maximal) event flexibility.

4. Compare the necessary amount of rolling stock per line for the timetable from the CONTRAVEL model with the given bound on rolling stock. If one line needs too much rolling stock, reduce $\epsilon$ by multiplying $\epsilon$ with a positive factor smaller than 1 and go step 3 .

5. Release timetable with event flexibility as reference timetable.

Iteration scheme 1 is clearly a heuristic scheme. The adjustment of the SI in step 1 and step 2 corresponds to the backward loop from 'capacity planning' to 'line planning, line concept' in Figure 1. This loop is not part of this paper but of ongoing research. A description of the state of the work on this loop can be found in Wüst et al. (2018a and 2018b) and Bütikofer et al. (2019).

The computed event flexibility in the reference timetable can be tested with respect to stability or feasibility on the micro level. If the flexibility is not satisfying, we may loop the CONTRAVEL model and adjust the weights $w_{e}$ in the objective function $f_{\text {flex }}$. This loop is also not part of this paper, but it is described in detail in Wüst et al. (2018b and 2019).

In step 2 and 4 we control the necessary amount of rolling stock. This is possible since we are including turnaround activities in our EAN according to Liebchen and Möhring (2007) (see section 3.3). 
2.4.2 Construction of a commercial timetable for periods with maintenance intervals

In this section we want to demonstrate how to adapt the iteration scheme 1 in order to generate a feasible timetable with event flexibility for maintenance intervals. During the respective maintenance interval, the scheduled trains in the temporary timetable should be as close to those in the reference timetable that it is possible to communicate only one 'commercial' timetable to the customers. This is positive from a customer perspective, but also from an operator's perspective since restoring the reference timetable after the maintenance interval has finished is easier in this case. In addition, the free capacity in the network can be used for additional services (e.g. freight trains) during the whole planning horizon.

Aim: Try to generate a commercial timetable with event flexibility feasible for no, one or several $(n)$ construction intervals (i.e. feasible for all scenarios with resource restrictions).

Input: (see iteration scheme 1) In addition:

- Infrastructure restrictions for all $n$ maintenance intervals

- Maximal time tolerance between event times of timetables with event flexibility of the single maintenance intervals. These event flexibility values represent the commercially tolerable variation of departure and arrival times during the planning horizon in contrast to operational event flexibility, which facilitates timetable feasibility at the microscopic level.

\section{Iteration scheme 2}

1. Start with a first construction interval: Compute a timetable with event flexibility for this construction interval with the help iteration scheme 1.

2. For each line take the passing times at the station nodes of the disaggregated network (see section 2.3) and add them to the SI with the expected tolerance time from the input. The remaining construction intervals will be computed with this adapted SI.

3. Compute the timetable with event flexibility for all construction intervals with the adaptations of the SI from step 2 and with the help iteration scheme 1.

4. Release timetables with event flexibility as interval timetables.

Iteration scheme 2 is again a heuristic scheme. As in iteration scheme 1, potential backward loops are not part of this paper (see comments in section 2.4.1).

In our case study (see section 3) we construct a timetable with event flexibility for each construction interval in the given timetable period. In practice, at every station and for every line the earliest departure and the latest arrival (with respect to all construction intervals) should be communicated as 'commercial' timetable to the customers.

\subsection{Generation of timetables based on a standard planning tool}

One of the main goals of the applied research project with SBB was to make the algorithmic timetable generation based on the proposed TCFPESP-method available to practitioners. Therefore, the generic configuration of any timetabling scenario 
should be possible, using a standard timetabling system such as "Viriato", which is in use at SBB for service planning (see Viriato Info Folder, 2018). All kinds of relevant timetabling information like line and infrastructure data attributes can be entered easily in the appropriate masks (e.g. track connectivity data such as route exclusions between section and station tracks). See the Viriato mask in Figure 7 for an example of the track configuration of an operation point and its neighbouring sections. For more detailed information we refer to the Viriato User Manual (Viriato, 2016).

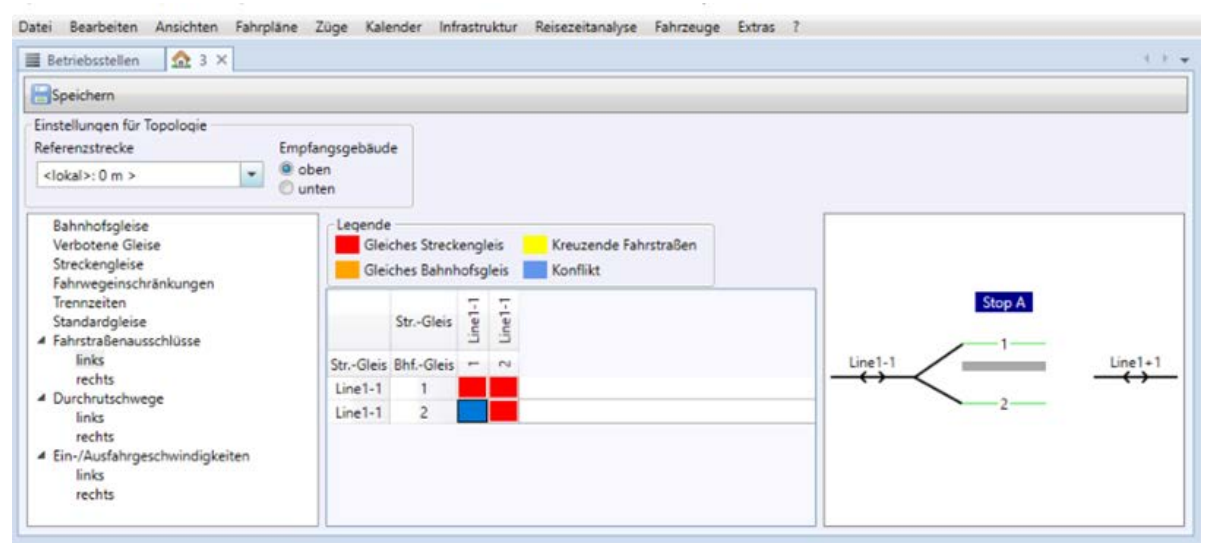

Figure 7: Viriato editor mask for entering timetabling configuration data attributes. The mask shows an example of track connectivity on one side of an operation point. Connectivity of station tracks and neighbouring section tracks as well as potential conflicts can be entered and configured using appropriate data masks.

\section{Case study 'Kerenzerberg'}

The selection of a scenario, suitable for testing the IP use case and our proposed timetabling tool was done based on proposals from practitioners. The corridor of 'Kerenzerberg' (see Figure 8) is well suited for a case study because it concerns important lines of the SBB network. On the other hand, the double-track corridor is separated from the rest of the network by two major stations at each side. The double-track section contains several stop stations and one single-track section close to one side of the corridor. This is the reference scenario and will be described in section 3.4. In one IP-scenario the maintenance interval concerns an extension of this single-track section. In a second IP-scenario the maintenance interval concerns a section at the opposite side of the corridor. The handling of these IP-scenarios will be described in section 3.5 .

We start with the description of mesoscopic infrastructure and SI on our test sector. According to the IP business requirements (see section 2.1), the SI is the result of the planning steps 'line planning, line concept' and 'traffic planning' (see Figure 1 in 2.1 for details) and is maintained in Viriato and the ZHAW planning tool. For the purpose of our case study, we adapted the existing SI for the timetable of 2018 in such a way that we are able to proof that we can handle the basic IP requirements with the 
proposed IP use case and the iteration schemes 1 and 2 for computer aided timetable generation.

\subsection{Description of the infrastructure}

The infrastructure between Ziegelbrücke and Sargans under normal operations is summarized in the following table. The infrastructure table is maintained in Viriato (see section 2).

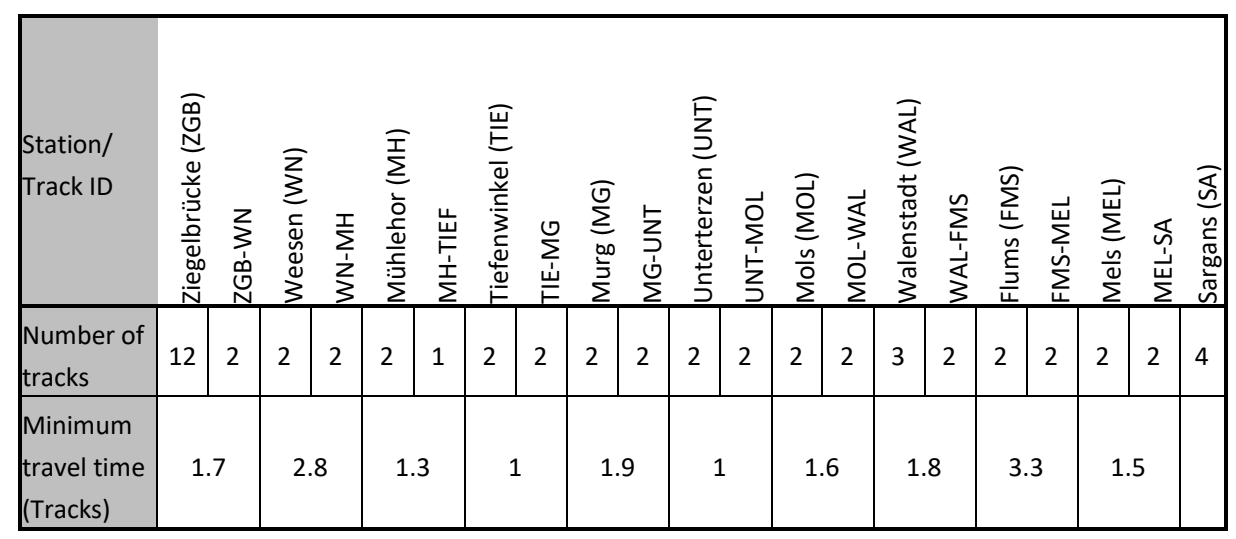

Table 1: Infrastructure data of the sector ZGB-SA. 'Number of tracks' indicates the number of tracks at stations (station name abbreviations in brackets) and in sections between stations (pair of neighbouring station abbreviations). 'Minimum trip time' indicates the maximum of the train and track specific technical trip times between station coordinates in minutes.

In the first row, we describe the stations (e.g. ZGB) and tracks (e.g. ZGB-MH). We see in Table 1 that there are always at least two tracks available, except between Tiefenwinkel and Mühlehorn, where only one track is available. Minimum trip times are derived from technical restrictions of the tracks.

\subsection{Network Segmentation}

In order to generate a traffic plan with capacity time bands, we have to segment the railway network into the relevant perimeter as explained in section 2.4. The SI in the next section is also adapted to the segmented network. We illustrate the network related to our case study 'Kerenzerberg' in Figure 8. 


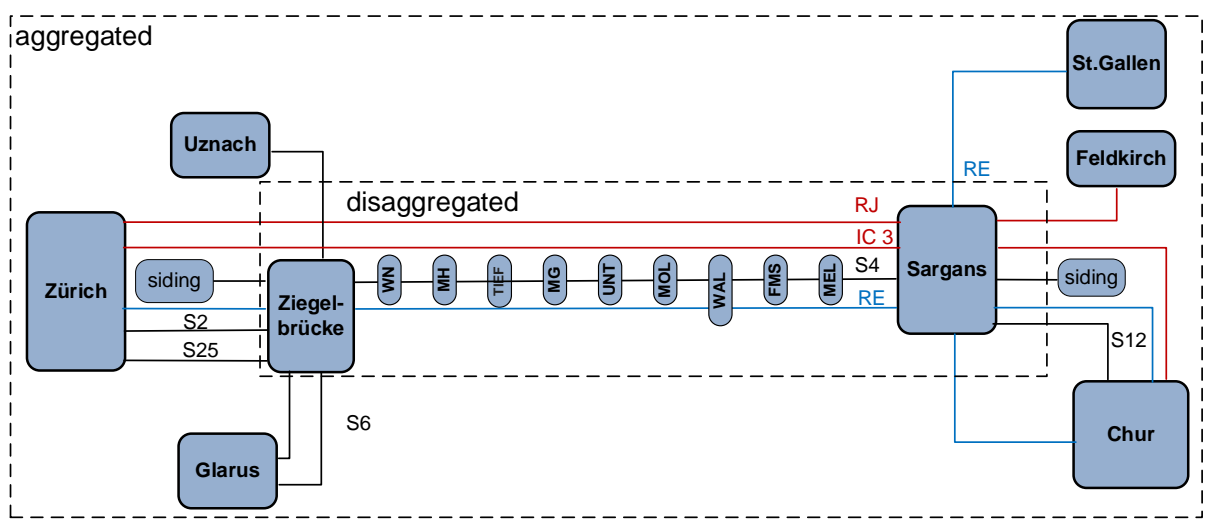

Figure 8: Network of the case study Kerenzerberg. A disaggregated subnetwork containing the relevant infrastructure segments at mesoscopic level and an aggregated subnetwork, representing simplified infrastructure on the macroscopic level.

As the planned construction or maintenance work for our test scenario is located on the network section between Tiefenwinkel and Mels, we decided to use the corridor Ziegelbrücke-Sargans as the disaggregated partition of the test network, where we will generate a detailed timetable (see section 2.4). The western part of Ziegelbrücke is aggregated, i.e. we introduced the nodes Uznach, Zürich, Glarus and a siding of Ziegelbrücke and connecting tracks. The aggregated network will be used to maintain vehicle circulation (e.g. turnarounds) aspects of lines and to model connections to tangent lines (see the description of SI in the next section). The eastern part of Sargans is also aggregated. We introduced the nodes St.Gallen, Feldkirch, Chur and a siding of Sargans. In the aggregated network we assume to have enough track capacity. Ziegelbrücke and Sargans can be considered as local hubs and represent the boundary nodes of the disaggregated network partition (see section 2.3). At these stations the timetable has to account for passenger transfers between lines. Technically spoken, these transfer requirements result in connections constraints in our TCFPESP-model.

\subsection{Description of Service Intention}

In section 2 we explained that the SI is our main data structure and is maintained in Viriato and the ZHAW planning tool. The SI contains all the information needed to configure the EAN and the TCFPESP model (see sections 2.2.2 and 2.2.3). We start with the lines considered. As mentioned before, our SI-lines represent an adaption of the lines in the corresponding timetable 2018. To demonstrate the turnaround operations, we decided that the line S4 makes a turnaround in a siding next to Ziegelbrücke and Sargans, respectively. 


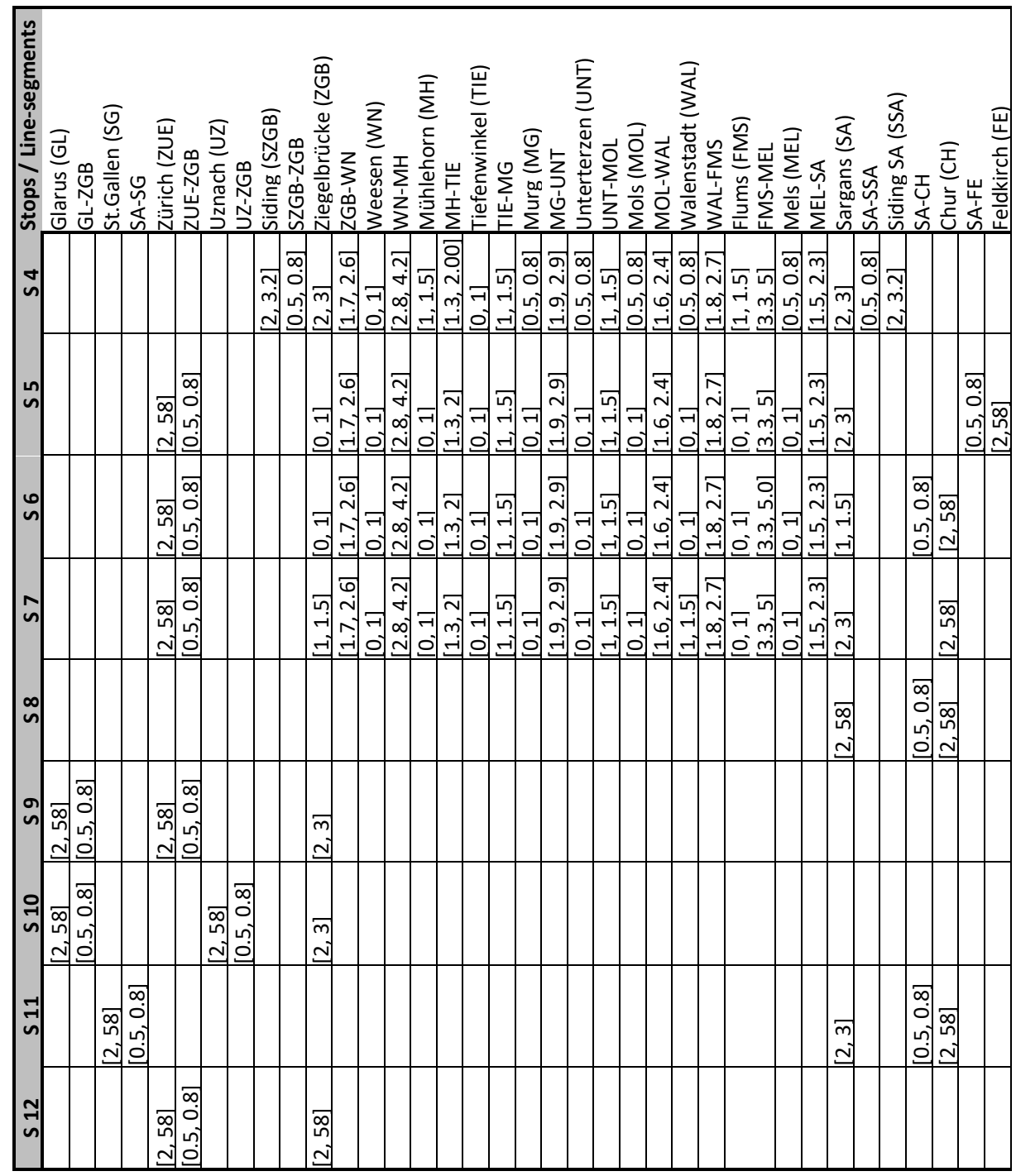

Table 2: Lines in the case study Kerenzerberg.

In Table 2 we summarized the upper and lower bound for dwell at every station ([Dlo, $\left.\left.\mathrm{D}^{\mathrm{up}}\right]\right)$ and trip time for every track ([TT $\left.\left.\mathrm{TT}_{\mathrm{lo}}, \mathrm{TT}^{\mathrm{up}}\right]\right)$. The routing can be derived from the entries in the table. A line visits all the stations and tracks from top to bottom and vice versa, where an upper and lower bound is given. Stations and tracks, which are not on the routing of a line, have no entry in corresponding field. In the first and the last station the lines perform a turnaround in the given interval ([TU $\left.\left.\mathrm{TU}_{\mathrm{lo}}, \mathrm{TU}^{\mathrm{up}}\right]\right)$. 
The minimum dwell $\mathrm{D}_{\text {lo }}$ and the minimum trip time $\mathrm{TT}_{\text {lo }}$ impose technical lower bounds. To compute the upper bounds $\mathrm{D}^{\text {up }}$ and $\mathrm{TT}^{\text {up }}$, we multiplied the lower bounds with 1.5. This reserve will be used to derive flexible plans with the TCFPESP model.

The turnaround times are computed according to the approach of Liebchen and Möhring (2007). The turnaround intervals are computed in such a way that a service with a minimal number of rolling stock is possible. In our case study, line S4 is operating with one rolling stock. The other lines operate with more than one rolling stock due to longer round-trip times. These bounds are not computed according to Liebchen and Möhring (2007), they are set manually. These lines can cross themselves in opposite directions (as it is in the real-world timetable). While the line information in Table 2 is mainly maintained in Viriato, the turnaround and connection times (see Table 3) are entered in the planning tool ZHAW.

The SI contains the following connections between the given lines:

\begin{tabular}{|c|c|c|c|c|c|c|c|c|c|c|c|c|}
\hline $\begin{array}{l}\text { Connection } \\
{\left[\mathrm{C}_{\mathrm{lo}}, \mathrm{C}^{\mathrm{up}}\right]} \\
\text { From/To } \\
\text { at station }\end{array}$ & $\begin{array}{l}\widetilde{\nwarrow} \\
\text { ஸे } \\
\text { 心 } \\
\text { 心 }\end{array}$ & 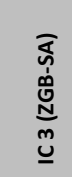 & 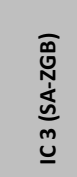 & 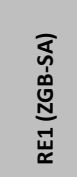 & 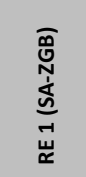 & 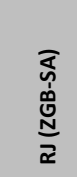 & 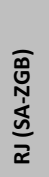 & 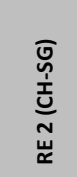 & 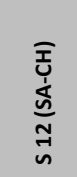 & 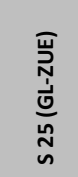 & $\begin{array}{l}\bar{J} \\
\stackrel{1}{ } \\
\stackrel{N}{N} \\
\stackrel{n}{n} \\
n\end{array}$ & 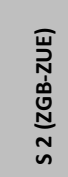 \\
\hline S4 & & & & & & & & & {$[1,15]$} & {$[1,15]$} & & {$[1,15]$} \\
\hline (ZGB-SA) & & & & & & & & & SA & SA & & ZGB \\
\hline S 4 & & & & & & & & & & {$[1,15]$} & & \\
\hline (SA-ZGB) & & & & & & & & & & ZGB & & \\
\hline IC 3 & & & & & {$[1,15]$} & {$[1,15]$} & & & & & & \\
\hline (SA-ZGB) & & & & & SA & SA & & & & & & \\
\hline RE 1 & & {$[1,15]$} & & & & & & {$[1,15]$} & & & & \\
\hline (ZGB-SA) & & SA & & & & & & SA & & & & \\
\hline RE 1 & & & & & & {$[1,15]$} & & & & & & \\
\hline (SA-ZGB) & & & & & & SA & & & & & & \\
\hline RE 2 & & & & & {$[1,15]$} & {$[1,15]$} & & & & & & \\
\hline (CH-SG) & & & & & SA & SA & & & & & & \\
\hline RE 2 & & & {$[1,15]$} & & {$[1,15]$} & {$[1,15]$} & & & & & & \\
\hline (SG-CH) & & & SA & & SA & SA & & & & & & \\
\hline S 6 & & & & {$[1,15]$} & & & & & & & & \\
\hline (GL- UZ) & & & & ZGB & & & & & & & & \\
\hline S 6 & & & & {$[1,15]$} & & & & & & & & \\
\hline (UZ-GL) & & & & ZGB & & & & & & & & \\
\hline S 12 & & & {$[1,15]$} & & {$[1,15]$} & {$[1,15]$} & & {$[1,15]$} & & & & \\
\hline (CH-SA) & & & SA & & SA & SA & & SA & & & & \\
\hline S 25 & {$[1,15]$} & & & & & & & & & & & \\
\hline (ZUE-GL) & ZGB & & & & & & & & & & & \\
\hline S2 & {$[1,15]$} & & & & & & & & & & {$[1,15]$} & \\
\hline (ZUE-ZGB) & ZGB & & & & & & & & & & ZGB & \\
\hline
\end{tabular}

Table 3: Connections in the case study Kerenzerberg

In Table 3 we find the implemented connections. The connections belong to the SI and are part of the output of the planning step 'line planning, line concept'. The connections should take place in the time interval $\left[\mathrm{C}_{\mathrm{lo}}, \mathrm{C}^{\text {up }}\right]$ from the line in the first col- 
umn to line in the corresponding column, e.g. there should be a connection from the line IC 3 (direction SA-ZGB) to line RE 1 (direction SA-ZGB) in Sargans with a minimum and maximum time of 1 and 15 minutes, respectively. The connection Table 3 is maintained in the ZHAW planning tool.

Furthermore, the SI contains:

- A time separation of the lines S4 (ZGB-SA) and RE 1 (ZGB-SA) of [20, 40] minutes in Ziegelbrücke. This should guarantee a frequent service for passengers travelling from Ziegelbrücke to Sargans.

- Trip time restrictions for the lines S4, IC 3, RE 1 and RJ between Sargans and Ziegelbrücke, i.e. trip times should be between 17 and 21 minutes for the IC 3, RE 1 and RJ. Line S4 is restricted to be between 20 and 29 minutes.

The time separation and the trip time restrictions are part of the output of the line planning step.

\subsection{Construction of a timetable with event flexibility for periods with normal operations at Kerenzerberg}

The models MINTRAVEL and CONTRAVEL are implemented in the algebraic modeling system GAMS (24.7.4). We use CPLEX (12.6.3) to solve these two MIP's. The stopping criterion of CPLEX was set to a relative gap (between the value of the actual best objective function and the lower bound value (MINTRAVEL) resp. upper bound value (CONTRAVEL)) of $10 \%$. In case of the MINTRAVEL objective function we set an absolute gap of 15 minutes in addition since the objective values can be close to 0. The computations were performed on Dell Latitude E6430 with an Intel 2.4 GHz quad core processor with $8 \mathrm{~GB}$ RAM.

In the case study Kerenzerberg we use the SI described in section 3.3. We have set the maximal flexibility $\delta_{\max }$ to 10 seconds and parameter $\epsilon$ to 0.5 . These values are based on experience of planning experts. The line S4 requires one rolling stock unit.

In step 1 of iteration scheme 1 the MINTRAVEL model results in a MIP with 23072 constraints and total 25629 variables (11401 integers). We could solve the MINTRAVEL model within 1272 seconds. The rolling stock of line S4 is 1. The CONTRAVEL model in step 3 results in a MIP with the same size as the MINTRAVEL model. We could solve the CONTRAVEL model within 1075 seconds. The rolling stock of line S4 is again 1. In total we have to solve the models MINTRAVEL and CONTRAVEL only one time. A reduction of $\epsilon$ in step 4 or an adjustment of SI in step 1 and 2 was not necessary.

We get the following reference timetable with event flexibility and resulting track allocations at the end of iteration scheme 1 . 
a

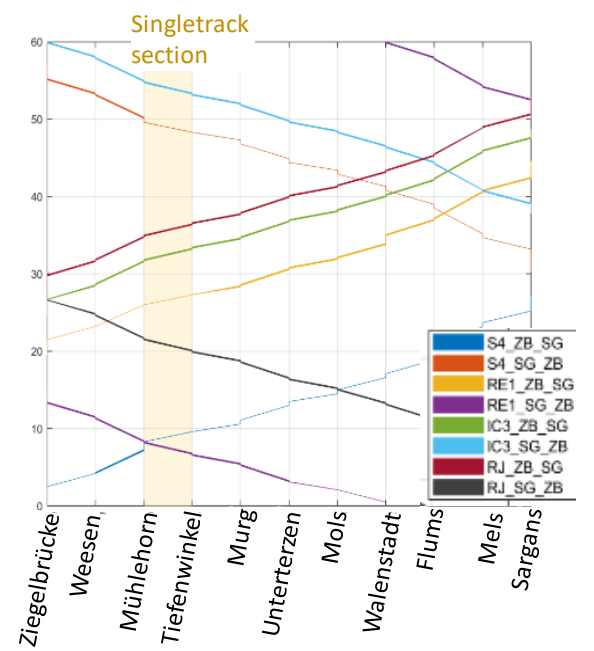

B

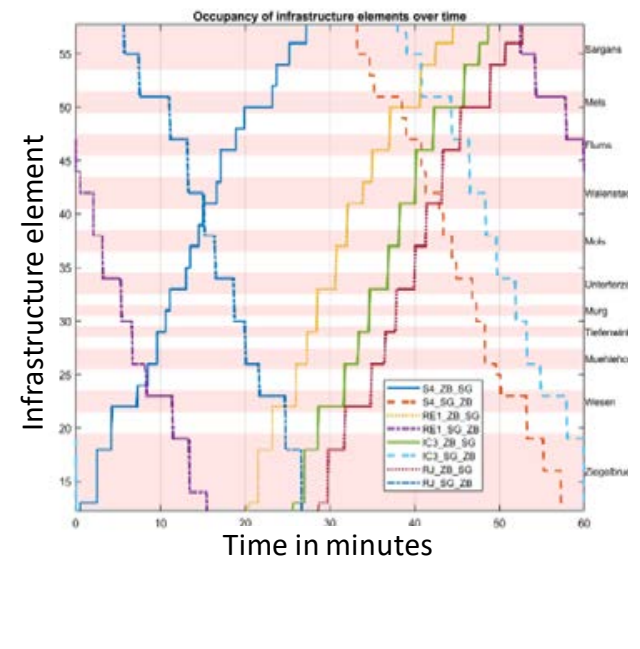

Figure 9: Reference timetable with event flexibility (a) and track assignment diagram (b) under normal operations. The track assignment diagram indicates which infrastructure element (enumerated track) is assigned to which train line during what time interval. Further explanations are given in the text.

Figure 9 illustrates the resulting reference timetable with the corresponding track allocation. As one can see, the SI is satisfied in general. Especially we can see that

- line S4 operates with one rolling stock as requested.

- the service of S4 and RE 1 is separated in Ziegelbrücke to guarantee smooth services to Sargans.

- the track choice method TCFPESP is able to generate a feasible track allocation on the mesoscopic infrastructure.

In Figure 9 one should note the fact that this reference timetable has crossings between Flums and Mels. It will not be feasible for the considered construction intervals in the next section 3.5.

\subsection{Construction of a commercial timetable for periods with maintenance intervals at Kerenzerberg}

In this section we want to demonstrate the application iteration scheme 2 from section 2.4.2. We consider two construction sites. The construction sites are between Tiefenwinkel and Unterterzen (construction site 1) resp. Flums and Mels (construction site 2). The construction intervals take place during our planning horizon but in different time windows. Only one track is available during the construction intervals on the affected corridors.

The computer infrastructure, software and parameters of iterations scheme 1 are the same as in section 3.4. Furthermore, we assume a maximal time tolerance of 6 minutes between the computed interval timetables (see section 2.4.2). 
In step 1 of iteration scheme 2 we started with construction interval 2 between Flums and Mels, since the single-track section is shorter than the one in construction interval 1 (see Figure 10). We could generate an interval timetable for construction site 2 without a reduction of $\epsilon$ or an adjustment of SI. In total we have to solve the models MINTRAVEL and CONTRAVEL only one time. The MINTRAVEL and the CONTRAVEL model results in a MIP with 23072 constraints and total 25581 variables (11353 integers). We could solve the MINTRAVEL resp. CONTRAVEL model within 1564 seconds resp. 1378 seconds. The models are little bit smaller in comparison to section 3.4 due to the reduced number of tracks during construction interval 2.

In step 2 of iteration scheme 2 we took the passing times from all lines in every station between Ziegelbrücke and Sargans from the interval timetable for construction site 2 and add them to the SI with the maximal tolerance of 6 minutes. For the construction interval 1 we allow therefore the lines to pass $+/-3$ minutes with respect to passing times from construction interval 2.

In step 3 of iteration scheme 2 we compute a timetable for construction interval 1 with the adapted SI from step 2. We could generate an interval timetable for construction site 1 without a reduction of $\epsilon$ or an adjustment of SI. In total we have to solve the models MINTRAVEL and CONTRAVEL only one time. The MINTRAVEL and the CONTRAVEL model results in a MIP with 23072 constraints and total 25586 variables (11385 integers). We could solve the MINTRAVEL resp. CONTRAVEL model within 19 seconds resp. 13 seconds. The faster running times are due to the adapted SI from step 2 (resp. the fixed passing times with a tolerance of $+/-3$ minutes).

a

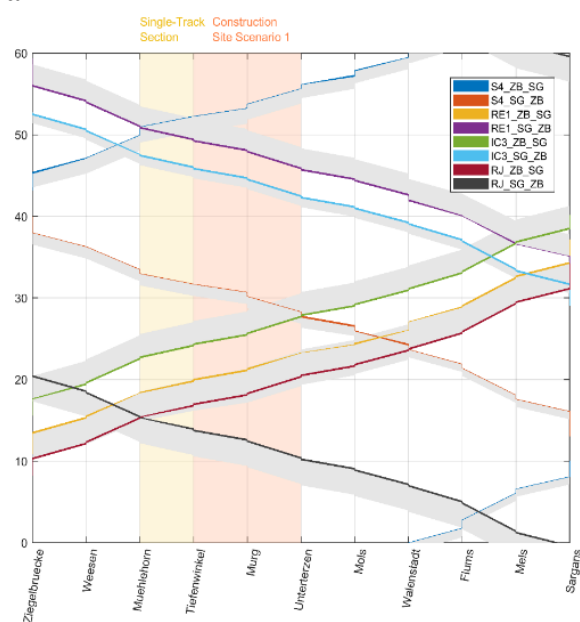

b

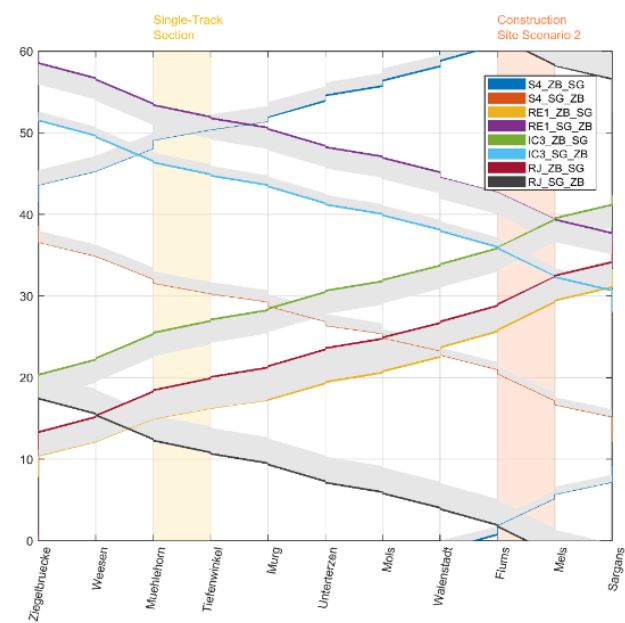

Figure 10: a) Timetable with event flexibility for construction interval 1, b) Timetable with event flexibility for construction interval 2. Both timetable scenarios (coloured lines are construction interval specific) are consolidated within one commercial Timetable (the grey bands contain the coloured lines). They are identical for both scenarios.

In step 4 of iteration scheme 2 we could release the interval timetables for construction site 1 and 2 and convert it into a 'commercial timetable'. 
In Figure 10 we see the interval timetables with event flexibility for both construction intervals. Due to iteration scheme 2 the timetables for the lines are at the lower or the upper boundary of the grey band. The grey band corresponds to the 'commercial' timetable. The timetable for construction interval 1 (Figure 10a) is not feasible for construction interval 2 (Figure 10b) and vice versa, e.g. line RJ and line S4 have a crossing between Flums and Mels during construction interval 1. It is worth mentioning that the order of line RJ and line RE1 from Ziegelbrücke to Sargans changes from construction interval 1 to 2 .

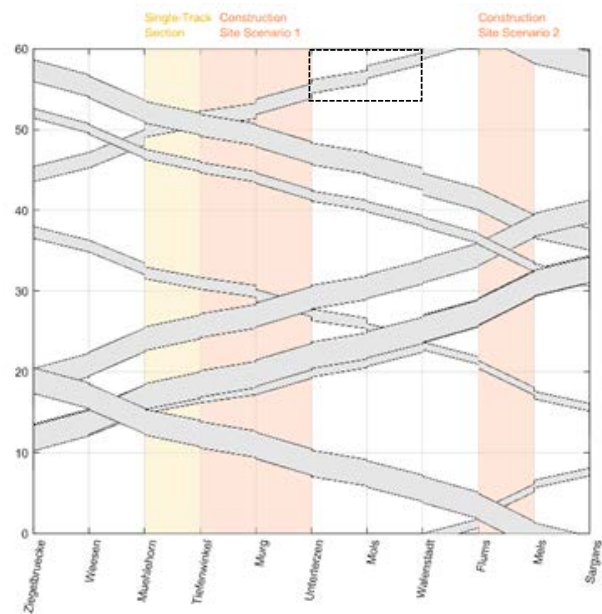

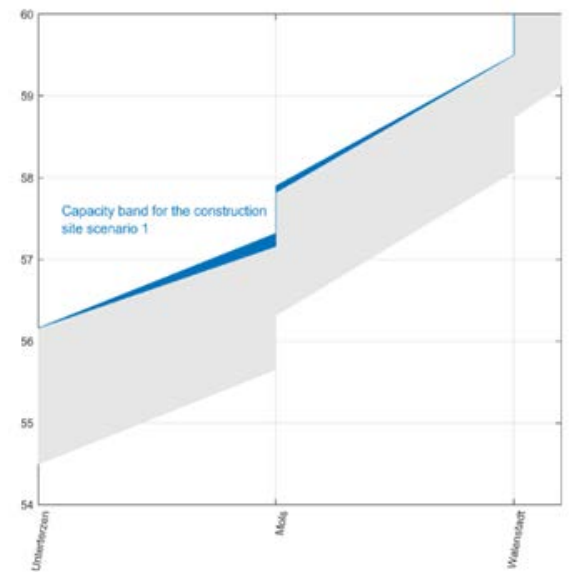

Figure 11: a) 'Commercial timetable', with boundaries of the grey time bands indicating earliest departure and latest arrival times b) Event flexibility of line S4 illustrated in blue color for the train slot between Unterterzen and Walenstadt. This example corresponds to the zoomed in area marked by the stipulated rectangle in a)

Figure 11a shows the 'commercial timetable' for the entire planning horizon covering both construction intervals. The departure times correspond to the lower boundary of the grey band and the arrival times to the upper boundary. During the planning horizon, we therefore always find a feasible timetable for all construction intervals. On the right (b) we see a detailed view of the timetable of the line S4 between Unterterzen and Walenstadt. The blue bands represent the event flexibility, e.g. during construction interval 1 we have around 10 seconds flexibility for the arrival and the departure in Mels.

Applying the iteration scheme 2 of section 2.4.2, we were thus able to generate one single commercial timetable with two similar but different capacity plans ('timetables with event flexibility'), which satisfies the SI during the entire planning horizon. That means that in practice the railway operator would have to communicate the commercial timetable to the passengers only once.

Hence, with the case study 'Kerenzerberg' showed that based on the SI and our TCFPESP-model, we were able to integrate operational stability (generating two different capacity plans) and passenger travel time aspects (finding one single commer- 
cial timetable with robust travel times as all transfers are guaranteed) in the proposed interval planning use case.

\section{Discussion and outlook}

\subsection{Summary}

We introduced and successfully applied the new timetabling model TCFPESP, which can be used to support timetable planners for generating train and vehicle schedules with track assignment. This model is based on an extension of the wellknown FPESP model and can be configured by using a standard schedule editor.

The use case and the TCFPESP model that we describe in section 2 are tested in a small-scale test and a real-world case study for IP in section 3 . The generation of the commercial timetable is achieved by an iterative execution of the IP use case for finding timetables with event flexibility for two different maintenance planning scenarios. We show how the concept of SI can be used to develop a customer timetable, which is valid during the complete timetable period. At the same time, it is now possible that two different construction or maintenance intervals with different locations can be planned during one single timetable period. This is of considerable practical relevance, especially with regard to the increasing number of intervals to be planned and executed under conditions of continued production of railway services.

\subsection{Outlook and future research}

If timetabling requirements turn out to be infeasible to be solved by TCFPESP, because, e.g., the given SI is not realizable on the respective railway infrastructure (a typical situation during construction intervals), this situation must be solved by a relaxation of the SI. This is indicated by the grey backward arrows in the planning process of the IP business requirements in section 2.1, showing that in this case one has to go back to previous planning steps and relax the SI. In a next research step, we want to find out, how the SI can be generated using standard line planning methods similar to those described by, e.g., Schöbel and Scholl (2006) or Friedrich et al. (2017). Our preliminary investigations show that these methods can generate SI configurations that take reduced resource availability (due to the fact that e.g. tracks are temporary out of service) into consideration. This research will help to make detailed specifications of data interfaces and service levels between TOC and IOC in case of IP and operational disruptions in real-time conditions.

Another aim of future research concerns the method for the utilization of timetable stability measures, such as cumulative delay impacts and cumulative delay sensitivity, obtained from timetable performance measurement for assigning event flexibility to improve timetable robustness (see Wüst et al. 2019 for preliminary results). With the outcome of this future research, we will be able to provide a detailed use case description of the iteration between the IP use case presented here and use cases for the assessment of timetable robustness. In that way, we expect to further improve the quality of TCFPESP results and contribute for speeding up and facilitating practical railway timetabling. 
The computational performance and scalability of PESP and FPESP based timetabling models has been investigated in detail (e.g. Herrigel 2015, Caimi et al. 2011b) and seems to be reasonable for medium size instances. The scalability of our TCFESP model to larger instances is subject to future research.

The use case presented in this paper refers to generating short-term timetable scenarios. The TCFPESP model is part of a planning framework, which is currently developed together with SBB and which also contains use cases for long-term process steps (Wüst et al. 2018b).

\section{Acknowledgements}

We highly acknowledge the whole SBB-team of SmartRail 4.0, Programm TMSPAS, for their encouraging support during the whole course of the project. We like to thank especially Thomas Wieland and Thomas Künzi for their inspiring ideas and the nice atmosphere, they knew to create.

This work was financially supported by the SBB Research Fund, c/o SBB Lab, Universität St.Gallen, Dufourstrasse 40a, 9000 St.Gallen.

\section{References}

Bešinović, N., Goverde, R.M.P., Quaglietta, E., Roberti, R., 2016. An integrated micromacro approach to robust railway timetabling. Transportation Research Part B: Methodological, vol. 87, pp. 14-32

Bütikofer, S., Frick, K., Köchli, J., Weber, C., 2019. Automatisierte Linienplanung im öffentlichen Verkehr, ETR Swiss / Wissen, accepted, Eurailpress.de

Caimi, G., 2009. Algorithmic decision support for train scheduling in a large and highly utilised railway network. Diss. ETH Zürich Nr. 18581, ETH Zürich, Zürich, Switzerland

Caimi, G., Laumanns, M., Schüpbach, K., Wörner, S., Fuchsberger, M., 2011a. The Periodic Service Intention as a Conceptual Frame for Generating Timetables with Partial Periodicity. Transportation Planning and Technology, vol. 34, issue 4, pp. 323-339

Caimi, G., Fuchsberger, M., Laumanns, M., Schüpbach, K., 2011b. Periodic railway timetabling with event flexibility. Networks, vol. 57, no. 1, pp. 3-18

Caprara A., Fischetti M., Toth P., 2002. Modeling and solving the train timetabling problem. Oper Res, vol. 50, pp. 851-861

de Fabris, S., Longo, G., Medeossi, G., Pesenti, R., 2014. Automatic generation of railway timetables based on a mesoscopic infrastructure model. Journal of Rail Transport Planning \& Management, vol. 4, pp. 2-13

Friedrich, M., Hartl, M., Schiewe, A., Schöbel, A., 2017. Integrating passengers’ assignment in cost-optimal line planning. Technical Report 2017-5, Preprint-Reihe, Institut für Numerische und Angewandte Mathematik, Georg-August Universität Göttingen. URL: http://num.math . uni-goettingen.de/preprints/files/2017-5.pdf

GAMS, 2018. General Algebraic Modeling System GAMS, GAMS Software GmbH, Fre- 
chen, Germany, URL: https : //WWW . gams . com/ (last accessed: 19 September 2018)

Hansen, I.A., Pachl., J. (eds.), 2008. Railway Timetable \& Traffic. Analysis - Modelling Simulation. Eurail Press, Hamburg, Germany

Herrigel, S., 2015. Algorithmic decision support for the construction of periodic railway timetables, Diss. ETH Zürich Nr. 22548, Department Bau, Umwelt und Geomatik, ETH Zürich, Zürich, Switzerland

Howald, P., Künzi, Th., Wild, P., Wieland, Th., 2017. Grobkonzept «Linienplanung» Version 0.7, 19.10.2017. SBB Smart Rail 4.0 TMS-PAS, project document repository

Liebchen, C., Möhring, R.H., 2007. The modeling power of the periodic event scheduling problem: Railway timetables - and beyond. In: Geraets, F., Kroon, L., Schöbel, A., Wagner, D., Zaroliagis, C. (eds.), Algorithmic Methods for Railway Optimization, Lecture Notes in Computer Science, vol. 4359, Springer, Berlin/Heidelberg, Germany, pp. 3-40

Peeters L., 2003. Cyclic railway timetable optimization. PhD thesis, Erasmus University, Rotterdam

$\mathrm{R}$, 2019. The $\mathrm{R}$ Project for Statistical Computing. URL: https://WWW.rproject . org/ (last accessed: 7 September 2018)

Schöbel, A., Scholl, S., 2006 Line planning with minimal travel time, 5th workshop on algorithmic methods and models for optimization of railways, Dagstuhl Seminar Proceedings 06901

Schlechte T., Borndörfer R., Erol B., Graffagnino T., Swarat E., 2011. Micro-macro transformation of railway networks. Journal of Rail Transport Planning \& Management, vol. 1, iss. 1 , pp. 38-48

Schlechte T., 2012. Railway Track Allocation: Models and Algorithms. PhD thesis, Technische Universität Berlin, Berlin

Schrijver A., Steenbeck A., 1994. Dienstregelingontwikkeling voor Railned (timetable construction for Railned). Rapport CADANS 1.0, C.W.I. Center for Mathematics and Computer Science, Amsterdam, In Dutch.

Serafini P., Ukovich W., 1989. A mathematical model for periodic scheduling problems. SIAM J Disc Math, vol. 2, pp. 550-581

SMA, 2018. Viriato - software for railways. Info Folder: URL: https://WWw . railsystemsengineering . com/RSE/documents/SMA\%20Vi riato\%20Brochure.pdf (last accessed: 7 September 2018)

SMA, 2016. Viriato - software for railways. User Manual (German): Viriato 8.9 Standard, Benutzerhandbuch

Wüst, R. M., Laube, F., Roos, S., Caimi, G., 2008. Sustainable Global Service Intention as objective for Controlling Railway Network Operations in Real Time, In: Proceedings of the WCRR 2008. Seoul

Wüst, R., Steiner, A., Gomez. C., 2017. Kundenfreundliche und robuste Ersatzfahrpläne während Bau- und Unterhaltsintervallen, ETR Swiss / Wissen, vol. 10, pp. 57-61, Eurailpress.de

Wüst, R., Bütikofer, S., Ess, Gomez, C., Steiner, A., Laumanns, M., Szabo, J., 2018a. Periodic timetabling with 'Track Choice'-PESP based on given line concepts and mesoscopic infrastructure. Operations Research 2018, Bruxelles, Conference proceedings 
Wüst, R. M., Bütikofer, S., Ess, S., Gomez, C., Steiner, A., 2018b. Development of a prototpe for the automated generation of timetable scenarios specified by the transport service intention. Abschlussbericht des Forschungsprojekt gefördert vom SBB Research Fund St. Gallen, https://imp-sbb-lab.unisg.ch/de/forschungsfonds-sbb-eng (last accessed: 15 August 2019)

Wüst, R., Bütikofer, S., Ess, S., Gomez, C., Steiner, A., Laumanns, M., Szabo, J., 2019. Improvement of maintenance timetable stability based on iteratively assigning event flexibility in FPESP. 8th International Conference on Railway Operations Modelling and Analysis, Norrköping, Sweden (accepted) 